\title{
Subjective cognitive decline in patients with migraine and its relationship with depression, anxiety, and sleep quality
}

\author{
Sun Hwa Lee ${ }^{1}$, Yeonwook Kang ${ }^{2}$ and Soo-Jin Cho ${ }^{*^{*}}$ (i)
}

\begin{abstract}
Background: Cognitive decline is a major concern in patients with migraine. Depression, anxiety, and/or poor sleep quality are well-known comorbidities of migraine, but available evidence on the subjective cognitive decline (SCD) is limited. This study aimed to investigate the presence and frequency of SCD and its relationship with anxiety, depression and sleep quality in patients with migraine.
\end{abstract}

Methods: We enrolled patients with migraine who scored within the normal range of the Korean-Mini Mental State Examination and the Korean-Montreal Cognitive Assessment. Using the Subjective Cognitive Decline Questionnaire (SCD-Q), participants with $\geq 7$ were assigned to the SCD group. The Headache Impact Test- 6 , Generalized Anxiety Disorder-7, Patient Health Questionnaire-9, and Pittsburgh Sleep Quality Index were used and analyzed between the two groups.

Results: A total of 188 patients with migraine, aged $38.1 \pm 9.9$ years, were enrolled. The mean SCD-Q score was $6.5 \pm 5.5$, and $44.7 \%$ of participants were identified as SCD. Migraineurs with SCD reported higher headache pain intensity and headache impact, as well as greater prevalence of anxiety, depression, reduced quality of sleep, and shorter sleep duration during weekdays compared to migraineurs without SCD. There were no significant differences in terms of age, sex, migraine type (chronic/episodic), medication, or sleep duration during weekends between the two groups. Upon multivariate logistic analysis adjusted for age, sex, headache characteristics, and psychological variables, depression was associated with increased risk of SCD (Odds ratio 1.31, 95\% confidence interval 1.16-1.49) and sleep duration during weekdays was associated with decreased risk of SCD (Odds ratio $0.66,95 \%$ confidence interval 0.44-0.97).

Conclusions: A non-negligible number of patients with migraine complained of SCD. Depression and short sleep duration during weekdays were related to SCD among adult migraineurs.

Keywords: Subjective cognitive decline, Migraine, Anxiety, Depression, Sleep

\section{Background}

Migraine is a common and disabling neurological disorder which leads to reduced quality of life and significant functional impairments [1]. In South Korea, a study investigating the prevalence of migraine over the course of 1 year reported that approximately 9.2\% of women and $2.9 \%$ of men suffer from the disease [2]. The burden of migraine is compounded by other

\footnotetext{
* Correspondence: dowonc@naver.com

'Department of Neurology, Hallym University Dongtan Sacred Heart Hospital, 7, Keunjaebong-gil, Hwaseong-si, Gyeonggi-do 445-907, South Korea Full list of author information is available at the end of the article
}

co-occurring disorders, including psychiatric disorders, sleep problems, epilepsy, and stroke [3]. Thus, it is important to identify manageable comorbidities for effective treatment of migraine.

There is contradictory evidence of the association between migraine and cognitive impairment. Some case-control studies reported that patients with migraine exhibit poor psychomotor speed [4], attention [5], and verbal memory [6] performance compared to non-migraineurs, even during postictal periods. Conversely, several longitudinal and cross-sectional studies have shown that migraine is not associated with 
cognitive dysfunction or decline and may even associate with better function or less decline [7-9]. Despite such inconsistent research findings on objective cognitive impairment, it is common that patients with migraine complain cognitive impairment in clinical practice. The perceptions of cognitive abilities are driven by comorbid symptoms rather than actual cognitive decline in patients with chronic pain. Therefore, cognitive problems in migraineurs is worthy to be approached from the assessment of complaints and its association with other comorbidities $[10,11]$.

Subjective cognitive decline (SCD), also known as "subjective memory impairment" in early studies, refers to any self-perceived or subjectively experienced worsening of cognitive function in the absence of impaired performance on cognitive tests [12]. SCD may represent the earliest symptoms of cognitive decline or preclinical Alzheimer's disease (AD) and is also associated with psychological factors such as depression and anxiety in cognitively healthy elders [13] and cerebrovascular disease [14-19]. Multiple factors seem to contribute to the development of SCD; however, most studies on SCD are on the elderly population. Some investigations on healthy adults report that memory complaints are common, regardless of age, and seem to be related to negative affect $[20,21]$. However, these studies focused only on "memory complaints" while difficulties in other cognitive domains, such as attention or executive function are also common in patients with migraine.

There is strong evidence indicating an association between migraine and several psychiatric conditions, such as mood and anxiety disorders [22, 23]. In a study using cluster analysis for patients with chronic migraine, patients with high levels of affective temperamental dysregulation showed a higher risk of suicidal behavior [24]. Recent studies also supported genome wide association between migraine and bipolar disorder, therefore, it is important to identify manageable psychiatric comorbidities for effective treatment and improving life quality among migraineurs $[25,26]$. However, an association between SCD and comorbid symptoms or pain severity has not yet been investigated with relevant cognitive tests among patients with migraine.

We hypothesized that the migraineurs with SCD would complain of more severe pain and exhibit greater psychiatric comorbidities than individuals with migraine without SCD. Thus, the aim of the present study was to investigate the presence and frequency of SCD in patients with migraine, as well as to analyze the association of SCD with clinical features and headache impact, anxiety, depression and quality of sleep.

\section{Methods \\ Participants}

The present study was based on a retrospective review of headache registry records. Records were of first visits for headache between January and November 2016 at the Department of Neurology Headache Clinic at the Hallym University Dongtan Sacred Heart Hospital. Inclusion criteria were as follows: 1) diagnosis of episodic migraine with or without aura, or diagnosis of chronic migraine, 2) completion of cognitive testing and questionnaires, and 3) normal performance on cognitive tests based on normative data, stratified by gender, age, and education level. Exclusion criteria were as follows: 1) diagnosis of primary headache disorder other than migraine, 2) secondary headache disorders other than medication-overuse headache or unclassified headache, 3) aged under 18 years or older than 65 years, 4) patients with migraine who were experiencing a severe attack, 5) refusal to perform cognitive testing or questionnaires (Fig. 1). The diagnosis of headache disorders was based on the beta version of the International Classification of Headache Disorder, 3rd edition [27]. Migraine with and without aura was considered as episodic migraine.

The participants completed questionnaires about SCD, psychological status, and headache impact. They also underwent cognitive screening tests for the evaluation of comorbid disorders. All participants were fully informed of the purpose and procedures of the study and were assured confidentiality.

The study protocol was reviewed and approved by the Institutional Review Board of the Hallym University Dongtan Sacred Heart Hospital. The Institutional Review Board allowed the process of informed consent to be waived due to the post-hoc analysis of collected clinical data.

\section{Assessments and procedures}

Demographic data such as age, sex, headache type (chronic/episodic and with aura/without aura/chronic/ probable), frequency of attack (per month), maximum pain duration (in hours), and number of tablets (per month) were collected and assessed when the participants first visited the clinic. Participants scored their subjective perception of average pain intensity on a Visual Analogue Scale (VAS) [28]. Headache impact was scored using the Headache Impact Test-6 (HIT-6), [29] which included six questions to measure headacherelated burden. The questions evaluated pain, social functioning, role functioning, vitality, cognitive functioning, and psychological distress. Each item was answered on a five-point scale $(6=$ never, $8=$ rarely, $10=$ sometimes, $11=$ very often, $13=$ always). Total scores ranged from 36 to 78 , and higher scores indicated greater impact. 


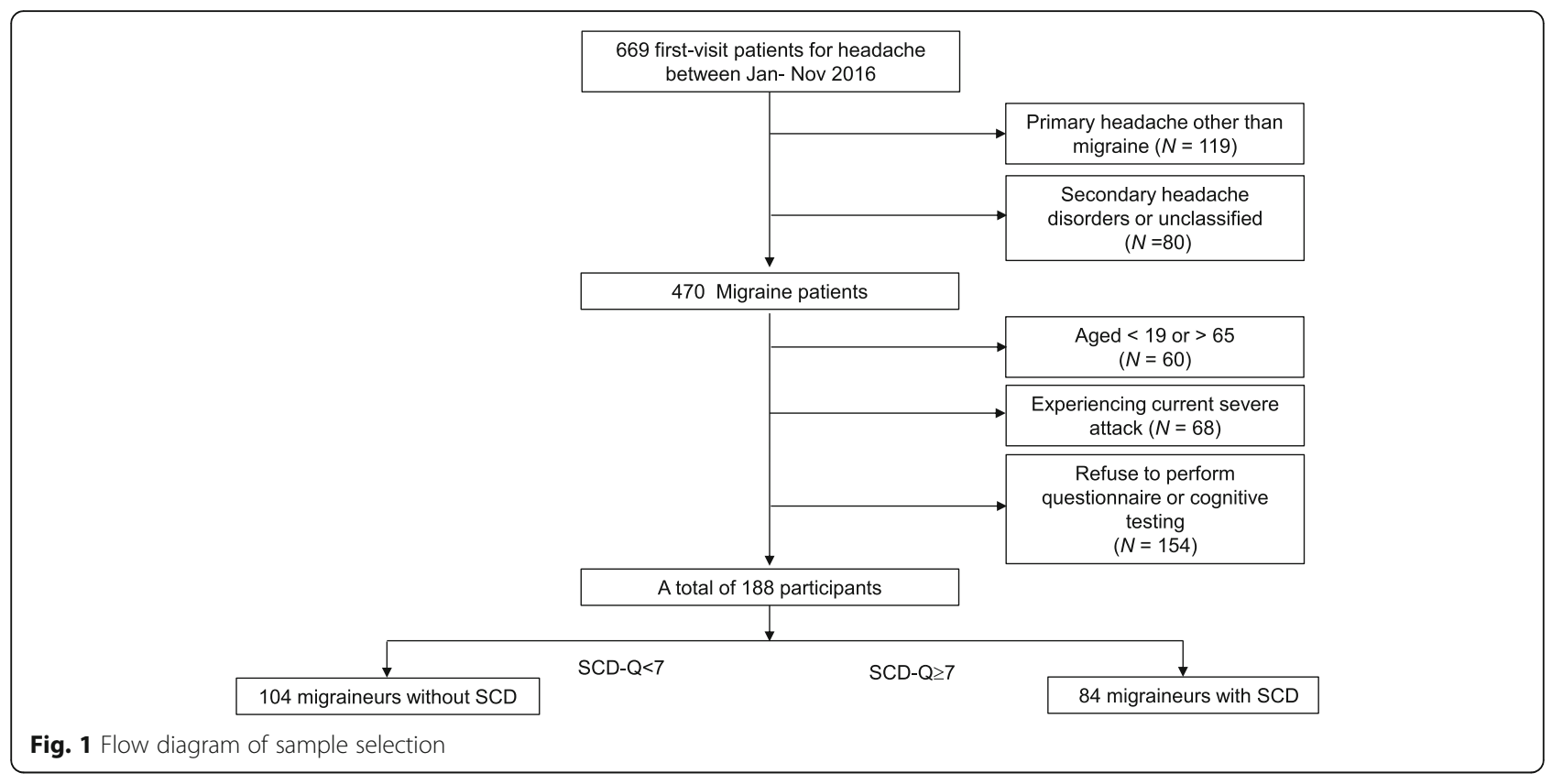

Two cognitive screening tests, the Korean-Mini Mental State Examination (K-MMSE) [30] and the Korean-Montreal Cognitive Assessment (K-MoCA) [31], were administered by a clinical neuropsychologist. The K-MMSE included items assessing orientation (5 points for time- and 5 points for place-orientation), memory (3 points for immediate- and 3 points for delayed-recall), serial subtractions ( 5 points), language ability ( 2 points for naming, 3 points for oral command comprehension, and 1 point each for repetition, reading, and writing), and visuospatial ability (1 point). The K-MoCA consisted of the following sequential items: alternate trail making (1 point), copying a cube (1 point) and drawing a clock (3 points) (to assess visuo-construction skills), naming (3 points), attention (6 points), sentence repetition (2 points), verbal fluency (1 point), abstraction ( 2 points), delayed recall ( 5 points), and orientation (6 points). An interval of 10 min was given between the two screening tests. Participants were administered the K-MMSE and $\mathrm{K}-\mathrm{MoCA}$ in counterbalanced fashion and the order of administration did not affect test performance. The total scores for both tests ranged from 0 to 30, and higher scores indicated greater cognitive function.

The SCD questionnaire (SCD-Q) [32] was used to assign participants to either the "no SCD group" or the "SCD group". The SCD-Q consisted of 24 items assessing three cognitive areas, including memory (11 items), language (6 items), and executive (7 items) domains. The response to each question was restricted to "yes/no" based on one's perceived decline in each item. The total SCD-Q score ranged from 0 to 24, with higher scores indicating greater subjective perception of one's cognitive decline over the past 2 years. The cutoff value was $\geq 7$.
The following self-report questionnaires were also administered to reveal clinical characteristics often complained by patients with migraine.

The Generalized Anxiety Disorder-7 (GAD-7), [33] which included seven items pertaining to the Diagnostic and Statistical Manual of Mental Disorders, 5th Edition (DSM-IV) criteria for a generalized anxiety disorder [34]. Each item was rated on a four-point scale, from 0 to $3(0=$ never, $1=$ several days, $2=$ more than half the time, and $3=$ nearly every day). Items were rated based on occurrence over the previous 2 weeks. The total scores ranged from 0 to 21 and the cutoff score was 5 [35].

The Patient Health Questionnaire-9 (PHQ-9) [36], which included nine items pertaining to the DSM-IV criteria for a Major Depressive Disorder (MDD) [34]. Each item was rated on a four-point scale, from 0 to 3 $(0=$ never, $1=$ several days, $2=$ more than half the time, and 3 = nearly every day). Items were rated based on occurrence over the previous 2 weeks. The total scores ranged from 0 to 27. A cutoff score of 7 was considered sensitive and specific to detect MDD in a patient population with migraine [37].

The Pittsburgh Sleep Quality Index (PSQI) [38] was used to measure the quality and patterns of sleep over the preceding 4 weeks. The PSQI differentiated "poor" from "good" sleep by measuring the following seven areas: subjective sleep quality, sleep latency, sleep duration, habitual sleep efficiency, sleep disturbances, use of sleeping medication, and daytime dysfunction. Scoring was based on a $0-3$ Likert scale and the sum of scores for these seven components yielded one global score ranged from 0 to 21 . A total score of $>5$ indicated 
poor subjective sleep quality. In addition to the PSQI, participants were also asked to record the average amount of sleep they achieved during weekdays and weekends.

\section{Data analysis}

Microsoft Excel 2010 and PASW statistics for Windows (version 18.0; SPSS Inc., Chicago, IL) were used for data analysis. Test results are reported as mean and standard deviations (SD) for normally distributed continuous variables. An independent-sample t-test with Levene's test for equal of variances was conducted for parametric data. Pearson's chi-square test was performed for categorical variables. Correlational analyses were performed using Spearman's rank correlation coefficient $\left(r_{s}\right)$. Multivariate logistic regression analysis was also conducted to investigate whether SCD may be predicted by anxiety, depression and sleep quality in patient with migraine.

\section{Results}

Among a total of 669 first-visit patients for headache between January to November 2016, 481 patients were excluded due to following criteria (Fig. 1): having primary or secondary headache disorders other than migraine $(N=199)$, age criteria $(N=60)$, experiencing severe pain $(N=68)$, and refuse to perform questionnaire or cognitive testing $(N=154)$. Among migraineurs, none was excluded due to abnormal range of performance on both screening tests. Amongst the total of 470 migraine patients, the age of included patients $(N=188$, mean age $=38.09 \pm 9.92)$ were significantly younger than the excluded patients $(N=282$, mean age $=40.68 \pm 14.43$, $p=0.032$ ), because those who were aged $<19$ or $>65$ were excluded. However, the percentage of gender did not differ between excluded versus included migraine patients $(p=0.610)$.
A total of 188 participants with migraine, aged $38.1 \pm 9.9$ years, were included. The mean SCD-Q score was $6.5(\mathrm{SD}=5.5)$, with 84 patients $(44.7 \%)$ scoring higher than 7. Thus, $44.7 \%$ of participants were diagnosed as SCD in this study. Among the 188 participants, $106(56.4 \%)$ scored higher than the cutoff on the GAD-7, 98 (52.1\%) scored higher than the cutoff on the PHQ-9, and 154 (81.9\%) scored higher than the cutoff on the PSQI.

Demographic and headache-related clinical data are shown in Table 1. An independent-sample t-test and Pearson's Chi-square test did not reveal differences between groups in terms of age, sex, migraine type, pain duration, and medication. However, significant differences were found for pain intensity on the VAS and headache impact on the HIT-6. There were no significant differences between groups on both cognitive screening tests - the K-MMSE and K-MoCA (Table 1). Moreover, no group differences were found in any subitems of both tests.

Of the 84 migraineurs with SCD, 56 (66.7\%) scored higher than the cutoff on the GAD-7, 59 (70.2\%) scored higher than the cutoff on the PHQ-9, and 73 (86.9\%) scored higher than the cutoff on the PSQI. Group differences between SCD and no-SCD groups in terms of anxiety, depression, and sleep quality are shown in Table 2 . Significant differences were found in anxiety (GAD-7), depression (PHQ-9), sleep quality (PSQI), and average sleep (in hours) during weekdays. However, there was no significant group difference in terms of the average amount of sleep achieved during weekends.

SCD-Q scores were correlated with the depression on the PHQ-9 $\left(r_{s}=0.49, p<0.001\right)$, anxiety on the GAD-7 $\left(r_{s}=0.34, p<0.001\right)$, sleep quality on the PSQI $\left(r_{s}=0.33\right.$, $p<0.001)$, sleep duration during weekdays $\left(r_{s}=-0.20\right.$, $p=0.007)$, VAS $\left(r_{s}=0.17, p=0.017\right)$, and the frequency

Table 1 Demographical and clinical characteristics of no-SCD versus SCD groups

\begin{tabular}{llll}
\hline & No-SCD $(n=104)$ & SCD $(n=84)$ & $39.1 \pm 10.0$ \\
\hline Age & $37.3 \pm 9.8$ & $24 / 60$ & 0.228 \\
Sex (male/female) & $24 / 80$ & $65 / 19$ & 0.390 \\
Episodic/chronic headache & $84 / 20$ & $8 / 58 / 18 / 0$ & 0.569 \\
Migraine types (with aura/without aura/chronic/probable) & $5 / 75 / 20 / 4$ & $10.2 \pm 7.2$ & 0.179 \\
Frequency of attacks per month & $8.9 \pm 8.1$ & $31.3 \pm 31.3$ & 0.236 \\
Maximum pain duration in hours & $35.3 \pm 54.0$ & $5.6 \pm 6.7$ & 0.542 \\
Number of tablets per month & $4.3 \pm 5.9$ & $7.2 \pm 1.5$ & 0.169 \\
Pain intensity on VAS & $6.6 \pm 1.8$ & $62.3 \pm 7.0$ & 0.024 \\
HIT-6 & $59.2 \pm 7.6$ & $28.9 \pm 1.6$ & 0.005 \\
K-MMSE & $29.0 \pm 1.5$ & $27.5 \pm 2.0$ & 0.566 \\
K-MoCA & $27.5 \pm 1.9$ & 0.966 \\
\hline
\end{tabular}

VAS Visual Analogue Scale, HIT-6 Headache Impact Test- 6 , SCD subjective cognitive decline, K-MMSE Korean-Mini Mental State Examination, K-MoCA Korean-Montreal Cognitive Assessment 
Table 2 Anxiety, depression, sleep quality, and average sleeping hours during weekdays and weekends of no-SCD versus SCD groups

\begin{tabular}{llll}
\hline & No-SCD $(n=104)$ & $\operatorname{SCD}(n=84)$ & $p$-value \\
\hline GAD-7 & $5.0 \pm 3.9$ & $8.2 \pm 5.5$ & $<0.001$ \\
PHQ-9 & $5.7 \pm 4.0$ & $10.4 \pm 5.6$ & $<.001$ \\
PSQI & $8.5 \pm 3.8$ & $10.6 \pm 4.3$ & $<0.001$ \\
Average Sleeping hours during weekdays & $6.5 \pm 1.1$ & $6.0 \pm 1.3$ & 0.008 \\
Average Sleeping hours during weekends & $7.6 \pm 1.5$ & $7.3 \pm 2.0$ & 0.335 \\
\hline
\end{tabular}

GAD-7 The Generalized Anxiety Disorder-7, PHQ-9 The Patient Health Questionnaire-9, PSQI The Pittsburgh Sleep Quality Index, SCD subjective cognitive decline

of headache attacks per month $\left(r_{s}=0.15, p=0.047\right)$. However, SCD-Q scores were not significantly correlated with the HIT-6, age, maximum pain duration, medication, sleep duration during weekends, nor cognitive screening test scores (data not shown).

Upon multivariate logistic analysis adjusted for age, sex, headache characteristics, and psychological variables, depression was associated with increased risk of SCD (Odds ratio 1.31, 95\% confidence interval 1.161.49) and sleep duration during weekdays was associated with decreased risk of SCD (Odds ratio 0.66, 95\% confidence interval 0.44-0.97) (Table 3). As the level of depression on PHQ-9 increases by 1 unit, the likelihood of migraineurs with SCD increases by 0.272 times $\left(\mathrm{b}=0.27\right.$, Wald $\left.\chi^{2}(1)=17.50, p<0.001\right)$. As the sleep

Table 3 Association of SCD with demographical and clinical variables: Multivariate logistic regression analyses

\begin{tabular}{lll}
\hline & OR (95\% Cl) & $p$-value \\
\hline Age & $1.03(0.99-1.08)$ & 0.131 \\
Sex (male/female) & $0.88(0.40-1.95)$ & 0.749 \\
Episodic/chronic headache & $0.97(0.17-5.43)$ & 0.968 \\
Migraine types (with aura/without aura/ & $0.73(0.35-1.51)$ & 0.392 \\
chronic/probable) & & \\
Frequency of attacks per month & $1.02(0.95-1.09)$ & 0.568 \\
Maximum pain duration in hours & $0.994(0.983-1.006)$ & 0.331 \\
Number of tablets per month & $1.02(0.95-1.09)$ & 0.590 \\
Pain intensity on VAS & $1.14(0.90-1.45)$ & 0.268 \\
HIT-6 & $1.00(0.94-1.07)$ & 0.887 \\
K-MMSE & $1.02(0.75-1.37)$ & 0.919 \\
K-MoCA & $1.09(0.85-1.38)$ & 0.507 \\
GAD-7 & $0.96(0.86-1.07)$ & 0.492 \\
PHQ-9 & $1.31(1.16-1.49)$ & $<0.001$ \\
PSQI & $0.92(0.82-1.03)$ & 0.161 \\
Average Sleeping hours during & $0.66(0.44-0.97)$ & 0.036 \\
weekdays & & \\
Average Sleeping hours during & $1.02(0.77-1.34)$ & 0.913 \\
weekends & & \\
\hline
\end{tabular}

VAS Visual Analogue Scale, HIT-6 Headache Impact Test-6, SCD subjective cognitive decline, K-MMSE Korean-Mini Mental State Examination, K-MoCA Korean-Montreal Cognitive Assessment, GAD-7 The Generalized Anxiety Disorder-7, PHQ-9 The Patient Health Questionnaire-9, PSQ/ The Pittsburgh Sleep Quality Index duration during weekdays increases by 1 unit, the likelihood of migraineurs with SCD lower by 0.423 times $\left(\mathrm{b}=-0.423\right.$, Wald $\left.\chi^{2}(1)=4.39, p=0.036\right)$.

\section{Discussion}

We investigated SCD amongst the patients with migraine using a standard questionnaire with relevant cognitive testing. The main findings of this study were 1) SCD is relatively common in adult patients with migraine, 2) migraineurs with SCD reported severe headache pain severity and headache impact, 3) migraineurs with SCD were more depressed and anxious, while experiencing lowered sleep quality and sleep duration during weekdays were shorter, 4) depression on the PHQ-9 and sleep duration during weekdays were associated with SCD after adjusting demographic variables, headache related variables and psychological variables.

Previous studies found that patients with migraine showed normal range of performance on both the MMSE [39] and MoCA [40]. Similarly, none were excluded due to abnormal performance on screening tests among migraineurs in this study. Considering the mean SCD-Q scores of $3.2(\mathrm{SD}=3.7)$ in normal controls in the previous study, the mean SCD-Q score of 6.5 $(\mathrm{SD}=5.5)$ in migraineurs was relatively high [32]. Not negligible number of young adult migraineurs seemed to complaint cognitive decline and, to our knowledge, this is the first report to identify SCD in adult patients with migraines. Moreover, the proportion of SCD (44.2\%) is comparable to common comorbidities of migraine such as poor quality of sleep (81.6\%), anxiety $(55.8 \%)$ and depression (52.1\%).

SCD in preclinical AD received particular interest on aging studies since longitudinal data support SCD as a risk factor for future cognitive decline as well as $\mathrm{AD}$ dementia $[14,17,41]$. A review study on SCD suggested that the proposed age of onset in studies of preclinical $\mathrm{AD}$ is 60 years or older despite the age at SCD onset was not defined as a core criterion [42]. Unlikely to SCD in elderly, there is no conclusive evidence for that SCD or perceived forgetfulness in young adult is a risk factor for future cognitive impairment or dementia [43]. In studies of healthy adults, memory complaints are frequent regardless of age; however, the type of complaint 
varies and may be related to negative affect throughout adulthood [20,21]. This study also suggested that SCD among migraineurs require more attention to psychological or sleep problem rather than close follow-up of cognitive decline.

Subjective pain is a common symptom in patients suffering from depression and in turn, chronic pain may trigger a depressive state [44, 45]. Interestingly, migraineurs with SCD complained of more severe difficulties in subjectively reported measures, such as pain intensity and headache impact, compared to migraineurs without SCD, but this association was not persistent on logistic analysis. This implies such sensitivity to perceived pain or subjective difficulties may be related to psychological impairments and/or sleep problem among migraineurs with SCD.

In line with previous studies, our data also revealed high levels of anxiety and depression in patients with migraine [46]. Not surprisingly, depression was significantly associated with SCD among migraineurs in this study. It is possible that SCD among migraineurs may either independently or conjointly account for the high levels of depression and anxiety. Moreover, biased perceptions of cognitive function may relate more to emotional state than objective ability, causing individuals to misinterpret or exaggerate problems by overestimating minor cognitive disruptions. According to Beck's cognitive theory of depression, depression is associated with a negative view of oneself, environment, and future [47]. Increasing the awareness patients with migraine have of possible misperceptions could help them understand their neurological and psychological state more objectively.

Similar to a previous study, more than half of the total patients with migraine that were evaluated in the current study complained of poor sleep quality [48]; this percentage was even higher $(86.9 \%)$ in migraineurs with SCD. This relationship may be explained by the high correlation between SCD-Q and PSQI scores. Indeed, a previous study revealed an association between perception of poor sleep quality and worse cognitive performance in healthy elderly individuals [49]. In the current study, the average amount of sleep achieved during weekdays for migraineurs with SCD was less than $6 \mathrm{~h}$. This value is less than the recommended duration of 7 to $8 \mathrm{~h}$ for adults [50]. It has been reported that migraineurs who routinely sleep $6 \mathrm{~h}$ per night exhibit more severe headache patterns and sleep complaints than migraineurs who slept longer [51]. Thus, short sleep duration during weekdays may account for the poor perception of cognitive function and sleep quality observed in the current study.

Several limitations to this study should be addressed. The main limitations of the study would be that it is based on retrospective review of headache registry records. Therefore, this study may be biased in its sampling procedure and we cannot rule out the possibility that individuals who perceived SCD were more inclined to agree to cognitive testing. However, variables in this study were collected prospectively according to the registry and the difference between excluded and included patients was not significant in demographical characteristics such as gender. In addition, we used brief screening tests (i.e., K-MMSE and K-MoCA) as cognitive measures. These were employed as it is difficult to recruit a substantial number of patients willing to undergo comprehensive neuropsychological tests in clinical settings. Although there were no group differences in any sub-items of both tests, more detailed cognitive tests may help reveal subtle cognitive characteristics that might not yet exceed age-, sex-, and education-adjusted normal ranges in migraineurs with SCD. Lastly, future studies should include a healthy control group to provide better standards for understanding migraineurs with SCD.

\section{Conclusions}

In conclusion, SCD seems relatively common in adult migraineurs in terms of both gender and migraine subtype. Migraineurs with SCD reported severe pain and impact of headache, were more depressed, anxious, perceived poorer sleep quality, and shorter sleep duration during weekdays than those without SCD. Depression and shorter sleep duration significantly associated to the presence of SCD in migraineurs after adjusting other variables.

\section{Abbreviations}

DSM-IV: Diagnostic and Statistical Manual of Mental Disorders5th Edition; GAD-7: Generalized Anxiety Disorder-7; HIT-6: Headache Impact Test-6; KMMSE: Korean-Mini Mental State Examination; K-MoCA: Korean-Montreal Cognitive Assessment; MDD: Major Depressive Disorder; PHQ-9: Patient Health Questionnaire-9; PSQI: Pittsburgh Sleep Quality Index; SCD: Subjective Cognitive Decline; SCD-Q: SCD questionnaire; VAS: Visual Analogue Scale

\section{Funding}

The authors received no financial support for the research, authorship, and/or publication of this article.

\section{Availability of data and materials}

The datasets supporting the conclusions of this article are included within the article and the supporting material.

\section{Authors' contributions \\ SHL, YK, SJC contributed to the conception and design, or analysis and interpretation of data. SHL, YK, SJC were involved in collection, interpretation, and statistical analysis of data. SHL, YK, SJC were involved in drafting the manuscript or revising it critically for important intellectual content. SHL, YK, SJC read and have given final approval of the version to be published. SJC agrees to be accountable for all aspects of the work and ensures that questions related to the accuracy or integrity of any part of the work are appropriately investigated and resolved. All authors read and approved the final manuscript.}

Ethics approval and consent to participate

The study protocol was reviewed and approved by the Institutional Review Board of the Hallym University Dongtan Sacred Heart Hospital. The process of informed consent was waived due to the post-hoc analysis of the collected 
clinical data. All participants were fully informed of the purpose and procedures of the cognitive testing and questionnaire and were assured confidentiality.

\section{Consent for publication}

The study protocol was reviewed and approved by the Institutional Review Board of the Hallym University Dongtan Sacred Heart Hospital. The Institutional Review Board allowed the process of informed consent to be waived due to the post-hoc analysis of collected clinical data. All participants were fully informed of the purpose and procedures of the cognitive testing and questionnaire and were assured confidentiality.

\section{Competing interests}

Sun Hwa Lee reports no conflict of interest.

Yeonwook Kang reports no conflict of interest.

Soo-Jin Cho was involved as a site investigator of multicenter trial sponsored by Otsuka Korea, Eli Lilly and Company, Korea BMS, and Eisai Korea, and received research support from Hallym University Research Fund 2016 and received lecture honoraria from Yuyu Pharmaceutical Company.

\section{Publisher's Note}

Springer Nature remains neutral with regard to jurisdictional claims in published maps and institutional affiliations.

\section{Author details}

'Department of Neurology, Hallym University Dongtan Sacred Heart Hospital, 7, Keunjaebong-gil, Hwaseong-si, Gyeonggi-do 445-907, South Korea.

${ }^{2}$ Department of Psychology, Hallym University, Chuncheon, Korea.

Received: 17 May 2017 Accepted: 4 July 2017

Published online: 25 July 2017

\section{References}

1. Vos T, Allen C, Arora M, Barber RM, Bhutta ZA, Brown A et al (2016) Global, regional, and national incidence, prevalence, and years lived with disability for 310 diseases and injuries, 1990-2015: a systematic analysis for the global burden of disease study 2015. Lancet 388(10053):1545-1602

2. Kim BK, Chu MK, Lee TG, Kim JM, Chung CS, Lee KS (2012) Prevalence and impact of migraine and tension-type headache in Korea. J Clin Neurol 8(3): 204-211. doi:10.3988/jen.2012.8.3.204

3. Breslau N, Rasmussen BK (2001) The impact of migraine epidemiology, risk factors, and co-morbidities. Neurology 56(suppl 1):S4-S12

4. Scherer P, Bauer H, Baum K (1997) Alternate finger tapping test in patients with migraine. Acta Neurol Scand 96(6):392-396

5. Evers S, Bauer B, Suhr B, Husstedt I, Grotemeyer K (1997) Cognitive processing in primary headache a study on event-related potentials. Neurology 48(1):108-113

6. Hooker WD, Raskin NH (1986) Neuropsychologic alterations in classic and common migraine. Arch Neurol 43(7):709-712

7. Jelicic M, Van Boxtel MP, Houx PJ, Jolles J (2000) Does migraine headache affect cognitive function in the elderly? Report from the Maastricht aging study (MAAS). Headache 40(9):715-719

8. Leijdekkers ML, Passchier J, Goudswaard P, Menges LJ, Orlebeke JF (1990) Migraine patients cognitively impaired? Headache 30(6):352-358

9. Kalaydjian A, Zandi P, Swartz K, Eaton W, Lyketsos C (2007) How migraines impact cognitive function findings from the Baltimore ECA. Neurology 68(17):1417-1424

10. McCracken LM, Iverson GL (2001) Predicting complaints of impaired cognitive functioning in patients with chronic pain. J Pain Symptom Manag 21(5):392-396

11. Roth RS, Geisser ME, Theisen-Goodvich M, Dixon PJ (2005) Cognitive complaints are associated with depression, fatigue, female sex, and pain catastrophizing in patients with chronic pain. Arch Phys Med Rehabil 86(6):1147-1154

12. Jessen F, Amariglio RE, van Boxtel M, Breteler M, Ceccaldi M, Chetelat G et al (2014) A conceptual framework for research on subjective cognitive decline in preclinical Alzheimer's disease. Alzheimers Dement 10(6):844-852 doi:10.1016/j.jalz.2014.01.001

13. Balash Y, Mordechovich M, Shabtai H, Giladi N, Gurevich T, Korczyn A (2013) Subjective memory complaints in elders: depression, anxiety, or cognitive decline? Acta Neurol Scand 127(5):344-350

14. Dufouil C, Fuhrer R, Alpérovitch A (2005) Subjective cognitive complaints and cognitive decline: consequence or predictor? The epidemiology of vascular aging study. J Ame Geriatr Soc 53(4):616-621
15. Wang Y, West JD, Flashman LA, Wishart HA, Santulli RB, Rabin LA et al (2012) Selective changes in white matter integrity in $\mathrm{MCl}$ and older adults with cognitive complaints. Biochim Biophys Acta 1822(3):423-430

16. Stewart R (2012) Subjective cognitive impairment. Curr Opin Psychiatry 25(6):445-450

17. Glodzik-Sobanska L, Reisberg B, De Santi S, Babb JS, Pirraglia E, Rich KE et al (2007) Subjective memory complaints: presence, severity and future outcome in normal older subjects. Dement Geriat Cogn Disord 24(3):177-184

18. Wang Y, Risacher SL, West JD, McDonald BC, MaGee TR, Farlow MR et al (2013) Altered default mode network connectivity in older adults with cognitive complaints and amnestic mild cognitive impairment. J Alzheumers Dis 35(4):751-760

19. Archer HA, Newson MA, Coulthard EJ (2015) Subjective memory complaints: symptoms and outcome in different research settings. J Alzheumers Dis 48(s1):S109-S114

20. Ginó S, Mendes T, Maroco J, Ribeiro F, Schmand BA, De Mendonça A et al (2010) Memory complaints are frequent but qualitatively different in young and elderly healthy people. Gerontology 56(3):272-277

21. Rowell SF, Green JS, Teachman BA, Salthouse TA (2016) Age does not matter: memory complaints are related to negative affect throughout adulthood. Aging Ment Health 20(12):1255-1263

22. Hamelsky SW, Lipton RB (2006) Psychiatric comorbidity of migraine. Headache 46(9):1327-1333

23. Buse DC, Silberstein SD, Manack AN, Papapetropoulos S, Lipton RB (2013) Psychiatric comorbidities of episodic and chronic migraine. J Neurol 260(8):1960-1969

24. Serafini G, Pompili M, Innamorati M, Gentile G, Borro M, Lamis DA et al (2012) Gene variants with suicidal risk in a sample of subjects with chronic migraine and affective temperamental dysregulation. Eur Rev Med Pharmacol Sci 16(10):1389-1398

25. Jacobsen KK, Nievergelt CM, Zayats T, Greenwood TA, Anttila V, Akiskal HS et al (2015) Genome wide association study identifies variants in NBEA associated with migraine in bipolar disorder. J Affect Disord 172:453-461

26. Oedegaard K, Greenwood T, Johansson S, Jacobsen K, Halmoy A, Fasmer O et al (2010) A genome-wide association study of bipolar disorder and comorbid migraine. Genes Brain Behav 9(7):673-680

27. Headache Classification Committee of the International Headache Society (IHS) (2013) The international classification of headache disorders, (beta version). Cephalalgia 33(9):629-808

28. Wewers ME, Lowe NK (1990) A critical review of visual analogue scales in the measurement of clinical phenomena. Res Nurs Health 13(4):227-236

29. Kosinski M, Bayliss M, Bjorner J, Ware J, Garber W, Batenhorst A et al (2003) A six-item short-form survey for measuring headache impact: the HIT-6 ${ }^{\mathrm{TM}}$. Qual Life Res 12(8):963-974

30. Kang Y, Na DL, Hahn S (1997) A validity study on the Korean mini-mental state examination (K-MMSE) in dementia patients. J Korean Neurol Assoc 15(2):300-308

31. Kang Y, Park J, Yu K, Lee B (2009) A reliability, validity, and normative study of the Korean-Montreal cognitive assessment (K-MoCA) as an instrument for screening of vascular cognitive impairment (VCI) Korean. J Clin Psychol 28:549-562

32. Rami L, Mollica MA, García-Sanchez C, Saldaña J, Sanchez B, Sala I et al (2014) The subjective cognitive decline questionnaire (SCD-Q): a validation study. J Alzheumers Dis 41(2):453-466

33. Spitzer RL, Kroenke K, Williams JB, Löwe B (2006) A brief measure for assessing generalized anxiety disorder: the GAD-7. Arch Intern Med 166(10):1092-1097

34. America Psychiatric Association (2013) Diagnostic and statistical manual of mental disorders (DSM-5 ${ }^{\oplus}$ ). (5th ed.). Washington: American Psychiatric Pub. pp. 155-233

35. Seo JG, Park SP (2015) Validation of the generalized anxiety disorder-7 (GAD-7) and GAD-2 in patients with migraine. J Headache Pain 16(1):97

36. Kroenke K, Spitzer RL (2002) The PHQ-9: a new depression diagnostic and severity measure. Psychiatr Ann 32(9):509-515

37. Seo J-G, Park S-P (2015) Validation of the patient health questionnaire-9 (PHQ-9) and PHQ-2 in patients with migraine. J Headache Pain 16(1):65

38. Buysse DJ, Reynolds CF, Monk TH, Berman SR, Kupfer DJ (1989) The Pittsburgh sleep quality index: a new instrument for psychiatric practice and research. Psychiatry Res 28(2):193-213

39. Wen K, Nguyen N, Hofman A, Ikram M, Franco O (2016) Migraine is associated with better cognition in the middle-aged and elderly: the Rotterdam study. Eur J Neurol 23(10):1510-1516

40. Santangelo G, Russo A, Trojano L, Falco F, Marcuccio L, Siciliano M et al (2016) Cognitive dysfunctions and psychological symptoms in migraine without aura: a cross-sectional study. J Headache Pain 17(1):76 
41. Reisberg B, Shulman MB, Torossian C, Leng L, Zhu W (2010) Outcome over seven years of healthy adults with and without subjective cognitive impairment. Alzheimers Dement 6(1):11-24

42. Jessen $\mathrm{F}$, Amariglio RE, Van Boxtel M, Breteler M, Ceccaldi M, Chételat G et al (2014) A conceptual framework for research on subjective cognitive decline in preclinical Alzheimer's disease. Alzheimers Dement 10(6):844-852

43. Mol ME, van Boxtel MP, Willems D, Verhey FR, Jolles J (2009) Subjective forgetfulness is associated with lower quality of life in middle-aged and young-old individuals: a 9-year follow-up in older participants from the Maastricht aging study. Aging Ment Health 13(5):699-705. doi:10.1080/ 13607860902845541

44. Katona C, Peveler R, Dowrick C, Wessely S, Feinmann C, Gask L et al (2005) Pain symptoms in depression: definition and clinical significance. Clin Med 5(4):390-395

45. Munce SE, Stewart DE (2007) Gender differences in depression and chronic pain conditions in a national epidemiologic survey. Psychosomatics 48(5):394-399

46. Lantéri-Minet M, Radat F, Chautard M-H, Lucas C (2005) Anxiety and depression associated with migraine: influence on migraine subjects' disability and quality of life, and acute migraine management. Pain 118(3):319-326

47. Beck AT (1967) Depression: Clinical, experimental, and theoretical aspects. Philadelphia: University of Pennsylvania Press

48. Seidel S, Hartl T, Weber M, Matterey S, Paul A, Riederer F et al (2009) Quality of sleep, fatigue and daytime sleepiness in migraine-a controlled study. Cephalalgia 29(6):662-669

49. Nebes RD, Buysse DJ, Halligan EM, Houck PR, Monk TH (2009) Self-reported sleep quality predicts poor cognitive performance in healthy older adults. J Gerontol A Biol Sci Med Sci 64(2):180-187. doi:10.1093/geronb/gbn037

50. Panel CC, Watson NF, Badr MS, Belenky G, Bliwise DL, Buxton OM et al (2015) Joint consensus statement of the American Academy of sleep medicine and Sleep Research Society on the recommended amount of sleep for a healthy adult: methodology and discussion. J Clinical Sleep Med 11(8):931-952

51. Kelman L, Rains JC (2005) Headache and sleep: examination of sleep patterns and complaints in a large clinical sample of migraineurs. Headache 45(7):904-910

\section{Submit your manuscript to a SpringerOpen ${ }^{\circ}$ journal and benefit from:}

- Convenient online submission

- Rigorous peer review

- Open access: articles freely available online

- High visibility within the field

- Retaining the copyright to your article

Submit your next manuscript at $\gg$ springeropen.com 